\title{
Evaluation of maxillary first molar rotation during direct miniscrew-supported maxillary canine retraction: a before-and-after study
}

\author{
Reem M. AlShazly ${ }^{1}$, Ahmad F. Almohammad ${ }^{1}$, Hend Salah ElSayed ${ }^{2 *}$ (i) and Amr Ragab EL-Beialy ${ }^{3}$
}

\begin{abstract}
Background: Extraction of the first premolar followed by canine retraction into the extraction space is a common treatment in orthodontics. Molar rotation occurs when they are used as anchorage units for the canine distalization. The aim of this single-arm clinical trial was to evaluate the maxillary first molar rotation during maxillary canine retraction with elastic power chains and direct miniscrew anchorage.

Results: The mean difference between pre-retraction and post-retraction molar rotation was $1.89^{\circ} \pm 0.6$ which was statistically insignificant.

Conclusion: The use of miniscrews for direct anchorage can eliminate unwanted rotation of the maxillary first molars during canine retraction.

Trial registration ClinicalTrials.gov, NCT04887974. Registered on May 6, 2021- Retrospectively registered https://clini caltrials.gov/ct2/show/NCT04887974?id=NCT04887974\&draw $=2 \&$ rank=1.
\end{abstract}

Keywords: Canine retraction, Molar rotation, Miniscrew, Temporary anchorage devices

\section{Background}

Class II, bimaxillary dentoalveolar protrusion and crowding are common malocclusions. The treatment often requires first premolar extraction with subsequent canine retraction. Canine retraction can be performed with sliding and non-sliding mechanics. Most commonly sliding mechanics is achieved using power chains or nickel titanium coil springs which produce intermittent forces or continuous forces, respectively.

Power chains produced similar rates of retraction to nickel titanium coil springs, and have the added advantage of being easier to use and cheaper in cost (Mohammed et al. 2018).

*Correspondence: hs.hafez@nrc.sci.eg

2 Orthodontics and Pediatric Dentistry Department, Oral and Dental Research Division, National Research Centre, 33 ElBuhouth St. Dokki, Giza, Egypt

Full list of author information is available at the end of the article
During canine retraction, tipping and rotation of the canines and first molars are common, if not controlled (Acar et al. 1999; Nightingale and Jones 2003).

The manner a tooth moves is dependent upon the nature of the force system. A force that does not pass through the center of resistance produces a rotational movement (Wahabuddin et al. 2015). Commonly, the first molars are used for anchorage control during canine retraction. The mesial force applied at the molar buccal surface leads to mesial tipping and mesio-palatal rotation of the crowns (Cousley and Sandler 2015; DiBiase 2015).

The use of miniscrews as anchorage modifies the force system and eliminates the molar rotational moment that is produced when the molars act as anchorage units.

The aim of this study is to evaluate maxillary first molar rotation during maxillary canine retraction with elastic power chains in first premolar extraction cases using miniscrews for direct anchorage. 


\section{Methods}

The study was performed between April 2016 and January 2017, in the outpatient clinic of the Orthodontic Department, Faculty of Oral and Dental Medicine, Future University in Egypt. The sample comprised of 20 quadrants in ten healthy orthodontic patients with Class II div 1 or bimaxillary dentoalveolar protrusion requiring maxillary first premolar extraction and have a full set of permanent teeth. The age range was 19-25 years. Patients who had received previous orthodontic treatment were excluded. Other exclusion criteria were active periodontal disease, systemic or bone diseases and medications that may affect bone metabolism. The ethical committee reviewed and approved the trial before the start date. The trial was registered in ClinicalTrials.gov on May 6, 2021; NCT04887974. The aim of the study was explained to the patients and those, who agreed to participate, signed a written informed consent.

Standard patient records were taken including study models, intra- and extra-oral photographs, panoramic and lateral cephalometric radiographs. The upper first and second molars were bonded or banded in cases with posterior deep bite. Canines and second premolars were bonded with bracket prescription $0.022^{\prime \prime}$ slot Roth system (American orthodontics brackets mini master Roth

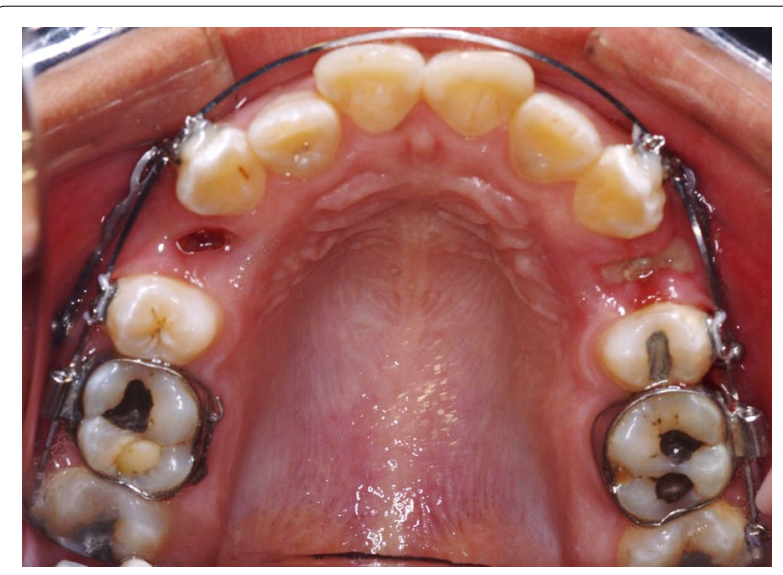

Fig. 1 Occlusal photograph $\left.0.022^{\prime \prime}\right)$. Brackets with vertical slots were bonded on canines while bypassing the four incisors.

Leveling and alignment of the arches were performed in sequential order until a $0.016^{\prime \prime} \times 0.022^{\prime \prime}$ stainless steel arch wire was reached. Anchorage was prepared by placing self-drilling miniscrews (Orthomed miniscrew (length; $8 \mathrm{~mm}$, Diameter; 1.2), Egypt) between second premolar and first molar. Patients were then referred for extraction of first premolars (Fig. 1). A cone beam computed tomography (CBCT) (Acteon $\mathrm{X}$-mind trium) was taken immediately before canine retraction. CBCT machine parameters were set to a moderate field of view.

Canine retraction was performed using a power chain (American orthodontics, USA; short power chain) on a $0.016^{\prime \prime} \times 0.022^{\prime \prime}$ stainless steel arch wire which was extended between the canine bracket's power arm $(8 \mathrm{~mm}$ in length from the horizontal slot) to the miniscrew with a force of $150 \mathrm{~g}$. The force magnitude was verified using a digital force gauge.

The power chain was activated every 4 weeks. After 6 months of canine retraction, the patients were referred for a second $\mathrm{CBCT}$.

$\mathrm{CBCT}$ images for each patient were analyzed. The landmarks, reference lines and planes are shown in Tables 1 and 2. The upper first molar rotation was measured as the angle between the molar horizontal axis and frontal Plane in the axial view (Fig. 2). The measurements were recorded in excel sheets.

\section{Data analysis}

The Shapiro-Wilk test was performed to test the normal distribution of the data. The change in molar rotation at 6 months was evaluated using the paired $t$ test.

\section{Results}

Means, standard deviations, and mean difference for the upper first molar rotation results are given in Table 3 . The mean difference was $1.89^{\circ} \pm 0.6$. There was no statistically significant difference $(P=0.1483)$ between the pre- and post-retraction rotation angle of the upper first molar.

Table 1 Landmarks

\begin{tabular}{ll}
\hline Landmark (abbreviation) & Definition \\
\hline 1. Anterior nasal spine (ANS) & Most anterior point on the tip of the anterior nasal spine \\
2. Posterior nasal spine (PNS) & Most posterior point on the hard palate at the tip of the posterior nasal spine \\
3. Incisive foramen (IF) & Most posterior point at the opening of the incisive foramen at the midline \\
& from the occlusal view \\
4. Upper first molar mesiobuccal cusp tip & The most occlusal point on the mesiobuccal cusp tip of the upper first molar \\
5. Upper first molar distobuccal cusp tip & The most occlusal point on the distobuccal cusp tip of the upper first molar \\
\hline
\end{tabular}


Table 2 Reference planes and line

\begin{tabular}{ll}
\hline Plane (abbreviation) & Definition \\
\hline 1. Midsagittal plane (MSP) & A plane passing through the ANS, PNS and IF \\
2. Horizontal plane (HP) & A plane passing through the ANS and the PNS. It is perpendicular to MSP \\
3. Frontal plane (FP): coronal & A plane passing through the IF. It is perpendicular to MSP and HP \\
4. Upper first molar horizontal axis & A line connecting the mesiobuccal and distobuccal first molar cusp tips \\
\hline
\end{tabular}

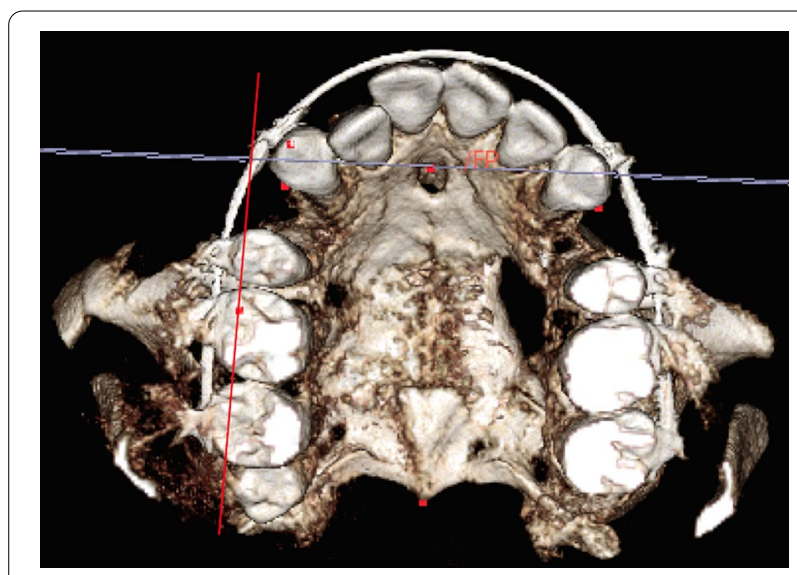

Fig. 2 Molar rotation measured to the constructed frontal plane

\section{Discussion}

This study is a single-arm clinical trial conducted to evaluate upper first molar rotation at 6 months of canine retraction with elastic power chains and miniscrews. Owman-Moll et al. (1995) found that power chains deliver intermittent forces and produce similar rates of retraction to nickel titanium coil springs. The optimum force for canine movement has not been identified in the orthodontic literature. However, a range of 100-200 g was suggested by Quinn and Yoshikawa (1985).

Although the molar rotation may have been assessed using dental models, the CBCT was used since other outcomes were being investigated as well. The advantage of $\mathrm{CBCT}$ imaging includes no superimposition of the right and left structures. CBCTs allow for a more accurate linear and angular measurement, better localization and identification of anatomical structures (Couceiro and Vilella 2010; Moreira et al. 2009). A substantial debate regarding the radiation dose of $\mathrm{CBCT}$ images exists. There are differences among the various CBCT machines for the effective radiation dose. Some panoramic $\mathrm{x}$-ray machines produce a higher dose than a single jaw CBCT images. The specifications used in this study are considered low. A brief scan time was chosen as prescribed by Feragalli et al. (2017). Furthermore, the participants in this trial are adults and are relatively less sensitive to ionizing radiation. We followed the ALARA principle to minimize the delivered ionizing radiation.

An important challenge during canine retraction is the maintenance of adequate intraoral anchorage. Anchorage loss is one of the main drawbacks during canine retraction. The movement of the molar into the extraction space that is intended for canine retraction is to be avoided in cases that require maximum anchorage. Temporary anchorage devices (TADs) were used to limit anchorage loss as described by Thiruvenkatachari et al. (2006), Antoszewska-Smith et al. (2017), and Becker et al. (2018). The best position for placement of the TADs were reported to be between the second premolar and first molar teeth, buccally (Schnelle et al. 2004; Fayed et al. 2010). The miniscrews were used as direct anchorage devices, leaving the first molars unloaded.

$\mathrm{CBCT}$ analysis showed mesio-palatal rotation of the first molars. The mean difference of rotation was clinically and statistically insignificant at $1.89^{\circ} \pm 0.6$. The amount of rotation in our study was less than the significant change reported by Aboalnaga (2017) at $2.21^{\circ} \pm 5.63$ and Rajcich and Sadowsky (1997) at $8.4^{\circ} \pm 5.6$. It was similar to $1.46^{\circ} \pm 1.46$ reported by Uzuner et al. (2015). In their study, despite the use of PG spring with anti-rotational moments, some molar rotation occurred.

Rajcich and Sadowsky (1997) found a positive correlation between molar anchorage loss and molar rotation.

Table 3 Maxillary first molar rotational change at 6 months of canine retraction using miniscrews for direct anchorage

\begin{tabular}{|c|c|c|c|c|c|c|c|c|c|c|}
\hline & $N$ & Mean & SD & SEM & Mean difference & SD & SED & $t$ & $d f$ & $P$ value \\
\hline Pre-retraction & 20 & 77.3910 & 6.7287 & 1.5046 & 1.8945 & 0.6 & 1.257 & 1.5067 & 19 & 0.1483 \\
\hline Post-retraction & 20 & 79.2855 & 5.9197 & 1.3237 & & & & & & \\
\hline
\end{tabular}

$\mathrm{N}$ : Sample size, SD: standard deviation, SED: standard error of difference 
Since they did not reinforce the anchorage units, the molars were allowed to move mesially. This high anchorage loss may explain the higher molar rotation. While the TADs used in our study and by Aboalnaga (2017) may have reduced the rotation by limiting anchorage loss of the molars.

The use of the miniscrews for direct anchorage eliminates the rotational moment created when first molars are used as anchorage units. This eliminates the need for anti-rotational mechanics during canine retraction with molar anchorage. They also limited the anchorage loss of molars which may cause additional molar rotation Rajcich and Sadowsky (1997).

\section{Conclusions}

There is no significant upper first molar rotation, at 6 months of canine retraction, using power chains and miniscrews for anchorage control.

\section{Abbreviations}

ANS: Anterior nasal spine; PNS: Posterior nasal spine; IF: Incisive foramen; MSP: Midsagittal plane; HP: Horizontal plane; FP: Frontal plane.

\section{Acknowledgements \\ Not applicable.}

\section{Authors' contributions}

RA and AA involved in investigation and writing-original draft preparation. HE involved in methodology, data curation, data monitoring and analysis, and writing-reviewing and editing. AE involved in methodology, visualization, and writing-reviewing and editing. All authors have read and approved the manuscript.

\section{Funding}

Self-funded.

\section{Availability of data and material}

The datasets used and/or analyzed during the current study are available from the corresponding author on reasonable request.

\section{Declarations}

\section{Ethics approval and consent to participate}

This trial was approved by the medical ethical committee at Future University in Egypt (Reference ID: 20153110). Patients who agreed to participate in this trial signed informed written consent.

\section{Consent for publication}

Not applicable.

\section{Competing interests}

The authors declare that they have no competing interests.

\section{Author details}

'Orthodontics and Dentofacial Orthopedics Department, Future University in Egypt, Cairo, Egypt. ${ }^{2}$ Orthodontics and Pediatric Dentistry Department, Oral and Dental Research Division, National Research Centre, 33 ElBuhouth St. Dokki, Giza, Egypt. ${ }^{3}$ Department of Orthodontics and Dentofacial Orthopedics, Faculty of Dentistry, Cairo University, Giza, Egypt.
Received: 19 May 2021 Accepted: 16 September 2021

Published online: 23 September 2021

\section{References}

Aboalnaga AA (2017) Effect of Micro-osteoperforation on the rate of canine retraction: a split mouth randomized controlled trial. Ph.D. thesis. Cairo University, Cairo, Egypt.

Acar A, Canyurek U, Kocaaga M, Erverdi N (1999) Continuous vs. discontinuous force application and root resorption. Angle Orthod 69:159-164

Antoszewska-Smith J, Sarul M, Lyczek J, Konopka T, Kawala B (2017) Effectiveness of orthodontic miniscrew implants in anchorage reinforcement during en-masse retraction: a systematic review and meta-analysis. Am J Orthod Dentofacial Orthop 151(3):440-455

Becker K, Pliska A, Busch C, Wilmes B, Wolf M, Drescher D (2018) Efficacy of orthodontic mini implants for en masse retraction in the maxilla: a systematic review and meta-analysis. Int J Implant Dent 4:35

Couceiro C, Vilella O (2010) 2D /3D Cone-Beam CT images or conventional radiography: which is more reliable ? Dent Press J Orthod. 15(5):40 e1-8

Cousley R, Sandler P (2015) Advances in orthodontic anchorage with the use of mini-implant techniques. Br Dent J 218(3):E4

DiBiase A (2015) Advances in orthodontic anchorage with the use of miniimplant techniques. Br Dent J 218:178-179

Fayed M, Pazera P, Katsaros C (2010) Optimal sites for orthodontic mini-implant placement assessed by cone beam computed tomography. Angle Orthod 80(5):939-951

Feragalli B, Rampado O, Abate C, Macrì M (2017) Cone beam computed tomography for dental and maxillofacial imaging: technique improvement and low-dose protocols. Radiol Med 122(8):581-588

Mohammed H, Rizk MZ, Wafaie K, Almuzian M (2018) Effectiveness of nickeltitanium springs vs elastomeric chains in orthodontic space closure: a systematic review and meta-analysis. Orthod Craniofac Res 21:12-19

Moreira C, Sales M, Lopes P, Cavalcanti M, Paulo S (2009) Assessment of linear and angular measurements on three-dimensional cone-beam computed tomographic images. YMOE 108(3):430-436

Nightingale C, Jones SP (2003) A clinical investigation of force delivery systems for orthodontic space closure. J Orthod 30:229-236

Owman-Moll P, Kurol J, Lundgren D (1995) Continuous versus interrupted continuous orthodontic force related to early tooth movement and root resorption. Angle Orthod 65:392-395

Quinn R, Yoshikawa D (1985) A reassessment of force magnitude in orthodontics. Am J Orthod 88:252-260

Rajcich M, Sadowsky C (1997) Efficacy of intraarch mechanics using differential moments for achieving anchorage control in extraction cases. Am J Orthod Dentofacial Orthop 112:441-448

Schnelle M, Beck F, Jaynes R, Huja SS (2004) A radiographic evaluation of the availability of bone for placement of miniscrews. Angle Orthod 74(6):832-837

Thiruvenkatachari B, Pavithranand A, Rajasigamani K, Kyung H (2006) Comparison and measurement of the amount of anchorage loss of the molars with and without the use of implant anchorage during canine retraction. Am J Orthod Dentofacial Orthop 129:551-554

Uzuner F, Yücel E, Göfteci B, Gül A (2015) The effect of corticotomy on tooth movements during canine retraction. J Orthod Res 3:181-187

Wahabuddin S, Mascarenhas R, lqbal M, Husain A (2015) Clinical application of micro-implant anchorage in initial orthodontic retraction. J Oral Implantol 41:77-84

\section{Publisher's Note}

Springer Nature remains neutral with regard to jurisdictional claims in published maps and institutional affiliations. 